OPEN ACCESS

Edited by:

Renee Heffron,

University of Washington,

United States

Reviewed by:

Lisa Lazarus,

University of Manitoba, Canada

Kathryn Therese Mngadi,

Aurum Institute, South Africa

*Correspondence:

Barbara A. Friedland

bfriedland@popcouncil.org

tThese authors have contributed equally to this work and share first authorship

Specialty section:

This article was submitted to HIV and ST/S,

a section of the journal

Frontiers in Reproductive Health

Received: 19 March 2021

Accepted: 25 May 2021

Published: 23 June 2021

Citation:

Friedland BA, Mathur $S$ and Haddad LB (2021) The Promise of the Dual Prevention Pill: A Framework for Development and Introduction. Front. Reprod. Health 3:682689. doi: 10.3389/frph.2021.682689

\section{The Promise of the Dual Prevention Pill: A Framework for Development and Introduction}

\author{
Barbara A. Friedland ${ }^{1 \star \dagger}$, Sanyukta Mathur ${ }^{2 \dagger}$ and Lisa B. Haddad ${ }^{1}$ \\ ${ }^{1}$ Population Council, Center for Biomedical Research, New York, NY, United States, ${ }^{2}$ Population Council, HIV and AIDS \\ Program, Washington, DC, United States
}

Women of reproductive age need multipurpose prevention technology (MPT) products to address two overlapping health risks: unintended pregnancy and HIV. Currently, condoms are the only available MPT, however male condoms are not within the control of a woman, and the use of female condoms has been limited by low acceptability and cost. Oral pre-exposure prophylaxis (PrEP) is highly effective for HIV prevention, yet uptake and adherence among women have been low to date. Women globally need more options for HIV and pregnancy prevention. Several MPTs for simultaneous HIV and pregnancy prevention are in various stages of development and clinical testing, although most are many years away from market launch. A dual prevention pill (DPP), a daily oral pill combining oral contraceptives and PrEP, both of which are licensed, approved products in many low- and middle-income countries (LMIC), is likely to be the fastest route to getting an MPT product into the hands of women. The DPP is one option that could enhance method choice, particularly for women who are already using oral contraceptives. By leveraging the oral contraceptive market and reaching women currently using condoms or with an unmet need for contraception, the DPP has the potential to increase the uptake of PrEP. The successful rollout of the DPP will require careful consideration of user-, provider-, and product-centered factors during product development and introduction. Early attention to these interrelated factors can help ensure that the DPP has the ideal characteristics for maximum product acceptability, that effective and quality services are designed and implemented, and that users can make informed choices, demand the product, and use it effectively. The proposed framework outlines key considerations for the effective development and introduction of the DPP, which could also facilitate integration models for future MPTs.

Keywords: HIV prevention, multipurpose prevention technologies, PrEP-FP integration, integrated healthcare, oral contraceptives, PrEP, dual prevention pill, informed choice

\section{INTRODUCTION}

Women worldwide are confronted with two significant, overlapping health risks: unintended pregnancy and HIV/sexually transmitted infections (STIs). More than 218 million women in lowand middle-income countries (LMICs), including $26 \%$ of women in sub-Saharan Africa (SSA), have an unmet need for contraception (1). Although, significant advances have been made in HIV treatment and prevention over the last decade, HIV/AIDS continues to be a leading cause 
of death among women of reproductive age globally (2). Nearly 800,000 women aged 15 and above were newly infected with HIV in 2019 (2). In SSA, women and girls accounted for 59\% of all new HIV infections, and adolescent girls and young women (AGYW) aged 15-24 years old were twice as likely to be living with HIV compared to their male counterparts (2). Despite substantial efforts by the global health community to integrate HIV and family planning service delivery, given the simultaneous risks of HIV infection and unintended pregnancy (3-7), for most women in LMICs, these services remain siloed (8).

Multipurpose prevention technology (MPT) products offer the potential to integrate sexual and reproductive health services and meet the diverse health needs of women over their reproductive lifespans (9-13). A growing body of literature indicates that a majority of women would be more interested in using an HIV-prevention method that also prevents pregnancy since preventing unintended pregnancy is often their primary concern (14-16). In a recent study from South Africa, significantly more women were interested in using the SILCS diaphragm together with a vaginal microbicide as an MPT (68\%) vs. SILCS alone for contraception (17\%) or a microbicide alone for HIV prevention (14\%) (17). In the Share.Learn.Shape global internet survey, $83 \%$ of women preferred an HIV/STI prevention method that also prevented unintended pregnancy vs. a product for disease prevention alone (14). In the same survey, there was high interest in a range of MPT products, including ondemand, daily, and long-acting methods. Furthermore, family planning is more acceptable than disease prevention in many communities. For example, some participants in the ASPIRE trial evaluating the safety and efficacy of the dapivirine intravaginal ring (IVR) reported telling their partners that they were using a new contraceptive vs. a product for HIV prevention (18). Currently, condoms are the only available MPT, yet male condoms are not within the control of a woman, and many women risk gender-based violence by merely suggesting condom use (19). The uptake of female condoms has been limited by cost, access, and acceptability issues (including the objections of male partners) $(20,21)$. MPTs could help to overcome barriers to negotiating HIV prevention and adherence issues related to stigma and gender dynamics seen in trials of microbicides and oral pre-exposure prophylaxis (PrEP). Several MPTs for simultaneous HIV and pregnancy prevention are in various stages of development, however, most are likely to be many years away from market launch $(22,23)$. A dual prevention pill (DPP) containing PrEP co-formulated with a combined oral contraceptive (COC) is likely to be the fastest route to the introduction of a female-initiated MPT because PrEP and generic COCs are both licensed, marketed products that are widely available in many LMICs (24).

\section{THE PROMISE OF THE DPP}

We believe that the DPP could vastly increase the number of women protected by PrEP as well as potentially increase the number of women using contraception. In the family planning arena, COCs continue to be the first-choice method for many women, despite the availability of longer-acting formulations. COCs are currently used by 151 million women worldwide (25), and over 5 million women in 15 SSA countries with a significant HIV epidemic (26). Oral PrEP, however, has had poor uptake and adherence among women to date, despite being highly effective for HIV prevention (27-29). Stigma is often cited as a reason for non-use of PrEP (30-33); women fear being regarded as HIVpositive or promiscuous if they are seen taking Truvada ${ }^{\circledR}$, the same antiretroviral (ARV) drug that is used for HIV treatment. Many women have voiced concerns about the consequences PrEP will have on their sexual relationships, as PrEP use often signals mistrust and infidelity, which can potentially result in relationship dissolution or violence $(33,34)$. We hypothesize that the DPP has the potential to reduce the stigma associated with PrEP-only products by adding the justification of providing contraception, as many women find it easier to negotiate contraception vs. HIV/STI prevention with their partners or have a shared desire for pregnancy prevention. Furthermore, we believe women's motivation to prevent pregnancy may drive adherence to PrEP when combined in a DPP. We estimate that by leveraging the $\mathrm{COC}$ market and reaching women currently using condoms or with an unmet need for contraception, between 250,000 and 1.25 million women per year in 15 SSA countries might choose to switch to the DPP, which could increase the number of women using PrEP by up to 10 times (26).

\section{DPP DEVELOPMENT PATHWAY}

The first generation DPP in development combines the active pharmaceutical ingredients (APIs) in a generic COC $[150 \mathrm{mcg}$ levonorgestrel (LNG), $30 \mathrm{mcg}$ ethinyl estradiol (EE)] with the APIs in Truvada ${ }^{\circledR}[300 \mathrm{mg}$ tenofovir disoproxil fumarate (TDF), $200 \mathrm{mg}$ emtricitabine (FTC)] or generic equivalents (35). The DPP regimen is intended to align with a 21/7 COC regimen containing 21 tablets with active COCs and PrEP, and 7 tablets containing only PrEP (vs. placebo pills in current 21/7 COC regimens). Because PrEP and COCs are already licensed, marketed products, the development pathway (Figure 1) is streamlined, requiring only a bioequivalence (BE) study rather than long, expensive Phase 3 safety and efficacy trials. In a standard BE study, healthy volunteers are enrolled in a crossover design to compare and ensure that the pharmacokinetic profile of the new drug (the DPP in this case) matches that of the reference products (Truvada and COC). Ideally, the DPP will be marketed in blister packaging to look as similar as possible to a contraceptive regimen (recognizing that Truvada is a much larger tablet than any COC). Both Viatris and the Population Council are developing DPP formulations, with potential approval as early as 2023 . Given the short timeline for development, preparing for an introduction now is critical to maximizing the potential reach of the DPP.

\section{FRAMEWORK TO GUIDE DPP PRODUCT DEVELOPMENT AND INTRODUCTION}

The development of new technology in and of itself does not imply demand, access, or use (36). The social and structural context of product provision can have an outsized influence on 


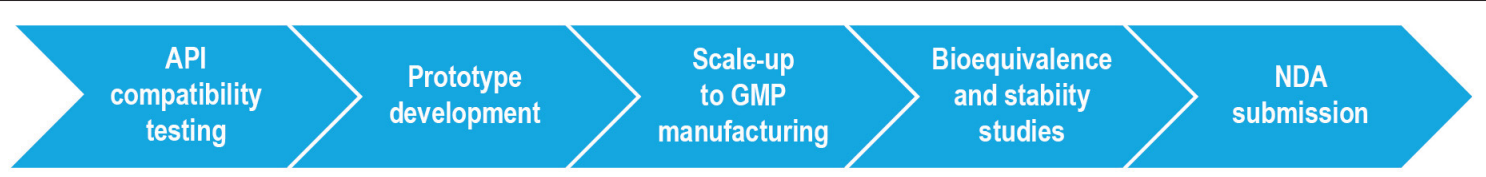

FIGURE 1 | DPP Development Pathway.

informed choice, product uptake, and effective and sustained use. Furthermore, there has been a growing recognition that enduser perspectives are important in the product development cycle and can help identify modifiable factors to inform formulation scientists about product attributes that may need optimization to enhance uptake, acceptability, and effective use (37-39). We propose a conceptual framework for DPP development and introduction in which user-, provider- and product-centered factors interact to influence user acceptability, intention-to-use, and, ultimately, product use (Figure 2). We used Ajzen's Theory of Planned Behavior (TPB) to guide the outcome of interest, the behavioral intention to use the DPP, as well as the framing of usercentered factors (40). We used previous frameworks on MPT development (10), PrEP introduction (41, 42), and contraceptive development and introduction $(36,43)$ to articulate the providerand product-centered factors. The user-, provider- and productrelated factors are situated within their socio-ecological levels, which interact to influence the intention of women to use the DPP.

In our framework, the aim is for the individual user to be enabled to make informed choices for HIV and pregnancy prevention options in choosing the DPP. According to the TPB, attitudes, subjective norms, and perceived behavioral control of an individual influence their behavioral intentions. In our framework, the characteristics of women, as well as their partnership dynamics, and their broader family and community context shape these intentions. The characteristics of women include life stage (such as age, marital status, and parity), knowledge (such as awareness of PrEP efficacy), perceived risk of acquiring HIV or having an unintended pregnancy, attitudes (about COCs/PrEP, perceived impact of acquiring HIV or having an unintended pregnancy), and experiences (such as contraceptive/PrEP use history). Partner dynamics that are likely to influence DPP use include the type of partner(s) that women have; the HIV status of the partner, risk behaviors, awareness, and approval of the DPP or other prevention products; interpersonal power and communication within relationships; and perceived or actual impact of the DPP on sexual activity and sexual pleasure (for women and their partners). Finally, in most settings, family and community context and prevailing social and gender norms (such as community perception of fertility, childbearing, and family size), perceptions of the DPP and other prevention products, norms about contraceptive and PrEP use, and HIVrelated stigma will likely influence an individual user's intention to use the DPP.

Provider-centered factors include elements that are important for ensuring the effective provision of quality services for the DPP. These include the healthcare providers and their knowledge about the DPP (such as indications, dosing regimen, counseling on side effects), attitudes, perspectives, and biases (such as frowning upon adolescent sexuality or favoring longacting contraceptive methods for their clients), and experiences (such as the prior provision of PrEP or contraception, counseling users on adherence). Additionally, the service delivery setting and broader health system infrastructure within which healthcare providers operate are likely to influence clientprovider interactions and the equitable access of women to the DPP. For example, it is critical to consider the type of training and support available to healthcare providers, their actual and perceived workload and responsibilities, product availability, and client flow at the service delivery setting. Similarly, at the health system level, product costs and financing, delivery platforms, task shifting (such as from clinicians to nurses or paramedical professionals, increased emphasis on self-care to lessen the burden on the health system), and demand generation for the DPP are just some of the key factors to consider for effective provision of the DPP.

Finally, we consider the ideal characteristics for DPP acceptability, centered around three key product attributes, the dosing regimen (daily), the APIs (including contraindications and side-effect profiles of the contraceptive hormones and ARVs), and the physical properties (tablet size). Layered on top of the product attributes are the policies and regulations (such as consent laws, regulatory approvals, and financing considerations) and the broader scientific landscape (such as product effectiveness relative to other products, medical screening and monitoring, and outcomes research) that are likely to influence DPP acceptability.

The three factors outlined in the framework are interrelated and dynamic; a shift in any single factor is likely to influence the intention to use the DPP by the individual user. For example, as next-generation DPPs are considered with different formulations or with different dosing regimens, service provision and user perspectives may also shift. Further, the actual use of the DPP and the subjective evaluation of that experience by an individual, the risks and benefits, will in turn influence behavioral intentions to continue using the DPP.

\section{DISCUSSION}

After decades of efforts to better integrate HIV and reproductive health services, the advent of novel MPTs that help women avoid unintended pregnancy and HIV may pave the way for providing more comprehensive care for individuals. The DPP offers one such potential for rapid development, introduction to the market, and expanding the method mix and choice for individual users. 


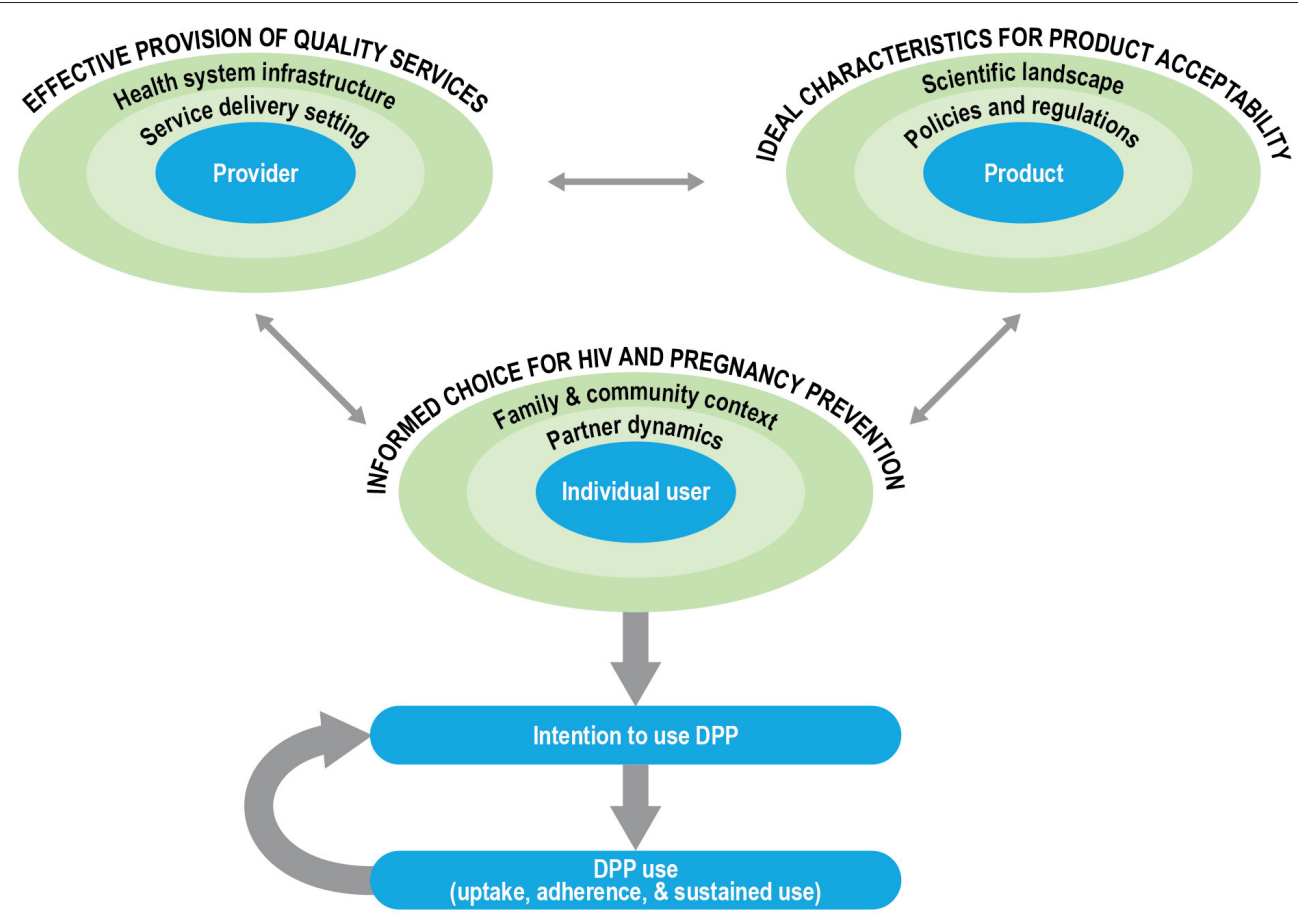

FIGURE 2 | Framework for DPP development and introduction.

At the same time, a systematic and coordinated approach to evidence generation is needed across product developers, socio-behavioral researchers, program developers, end-users, healthcare providers, and key stakeholders to maximize the potential impact of the DPP $(44,45)$. As we lay out in the proposed framework, critical questions must be assessed for user-, provider-, and product-centered factors to facilitate the effective, efficient, and equitable introduction of the DPP.

\section{End-User Research}

Evidence shows the importance of early engagement with potential end-users to identify facilitators and barriers to product acceptability, intention to use, product uptake, and effective use. For example, while it is assumed that combining HIV prevention with contraception will reduce the stigma associated with PrEP, empirical data will be needed to demonstrate that this is the case. Further, it will be important to ensure that rates of unintended pregnancy do not increase among COC users who switch to the DPP. For example, women taking COCs are advised to take two pills if they miss a dose; however, that is not the recommendation for PrEP and guidelines will need to be developed regarding missed doses. Counseling messages will need to be developed to position the DPP within the contraceptive method mix and ensure shared decision-making between providers and users for women to select the method that best matches their prevention priorities. Further, as with other PrEP products, women will need counseling on how to avoid STIs. Appropriate tools will need to be adapted and developed, including interactive client precounseling self-assessments to educate and counsel women about the anticipated side effects, risks, and benefits of the method to support effective and sustained use. Additional efforts will be needed to strategically engage male partners without diminishing women's autonomy $(33,46-48)$. End-user engagement will be key to inform demand creation, branding and marketing strategies, tools to support end-users, and implementation plans.

\section{Engaging Providers}

Providers play an instrumental role in influencing the demand, uptake, and effective use of new products. From the perspectives of reproductive health/family planning providers, the DPP or similar technologies introduced through family planning clinics could enhance integration but could also be a burden. The DPP may require additional HIV- or PrEP-related medical screening, testing, and monitoring that may be perceived to be outside of the scope of or burdensome to many family planning and primary healthcare providers. Concerns that the introduction of the DPP could influence client uptake of longacting contraceptive methods need to be mitigated by developing guidance for healthcare providers on how to support their clients to effectively meet their HIV and pregnancy prevention goals through a shared decision-making model of counseling and provision that maximizes client autonomy and informed choice to alleviate the potential for coercion. In particular, it will be important to explore the knowledge and attitudes of providers about the DPP, which will influence the access of women and the messages regarding the DPP. Mechanisms to effectively engage and support healthcare providers, including additional 
training and resources, within their eco-system will be critical to informing service delivery points about the need for the DPP.

\section{Policy and Regulatory Considerations}

Finally, to better understand how this new technology will be integrated within healthcare systems, further elucidation will be needed on product financing, market shaping and sizing, and value for money analyses. For the product developers, expanding beyond the current formulations of the DPP may help to further expand the market. Creating a co-formulated DPP with Descovy ${ }^{\circledR}$ [tenofovir alafenamide (TAF) and FTC], once it is approved for use as PrEP in heterosexual women, may be more appealing to women because of its smaller size. Alternative contraceptive regimens, such as extended cycles or progestin-only pills, may simplify the DPP regimen, offer more options, including for those with contraindications to estrogencontaining products (such as post-partum women) or those who desire amenorrhea $(49,50)$, and address challenges in counseling messages around missed doses for COCs vs. PrEP. Incorporating end-user preferences for the ideal DPP characteristics into the product development process is likely to enhance uptake.

\section{Conclusion}

We have laid out a broad scope of work, yet experience has shown us that asymmetric attention to any of these factors can lead to ineffective product uptake $(36,51)$. The proposed framework for guiding DPP development and introduction will continue to be important after the initial phases of product introduction to ensure that women can safely choose, access, and use the DPP. The DPP framework could serve as a model for the integration of future MPTs, including next-generation DPP products and other formulations like intravaginal rings and implants. Lessons learned from the DPP can pave the way for new technologies that best meet the needs of women, effectively destigmatize $\mathrm{HIV}$ as a general aspect of comprehensive reproductive health services, and lead to efficient integration of HIV and reproductive health services.

\section{REFERENCES}

1. Sully EA, Biddlecom A, Darroch JE, Riley T, Ashford LS, Lince-Deroche N, et al. Adding it up: Investing in Sexual and Reproductive Health 2019. Guttmacher Institute (2020). Available from: https://www.guttmacher.org/report/addingit-up-investing-in-sexual-reproductive-health-2019 (accessed MARCH 18, 2021).

2. UNAIDS. Global HIV\& AIDS statistics - 2020 Fact Sheet. UNAIDS. Available online at: https://www.unaids.org/en/resources/fact-sheet (accessed March 17, 2021).

3. Dehne KL, Snow R, O'Reilly KR. Integration of prevention and care of sexually transmitted infections with family planning services: what is the evidence for public health benefits? Bull World Health Organ. (2000) 78: 628-39.

4. Dickinson C, Attawell K, Druce N. Progress on scaling up integrated services for sexual and reproductive health and HIV. Bull World Health Organ. (2009) 87:846-51. doi: 10.2471/BLT.08.059279

5. Haberlen SA, Narasimhan M, Beres LK, Kennedy CE. Integration of family planning services into HIV care and treatment services: a systematic review:

\section{DATA AVAILABILITY STATEMENT}

The original contributions presented in the study are included in the article/supplementary material, further inquiries can be directed to the corresponding author/s.

\section{AUTHOR CONTRIBUTIONS}

BAF and SM conceptualized the DPP development and introduction framework and jointly drafted the manuscript. $\mathrm{LBH}$ is the senior author and provided strategic guidance on the development of the manuscript and the framework. All authors contributed to the writing and review of the manuscript.

\section{FUNDING}

This research was made possible by the support of the Children's Investment Fund Foundation (CIFF) (R-190303681) and the US National Institutes of Mental Health (NIMH) (1R34MH119982-01).

\section{ACKNOWLEDGMENTS}

We are grateful to our Population Council colleagues for their support of the DPP development work, in particular, Dr. George Creasy for spearheading the development of the DPP, Drs. Nanlesta Pilgrim and Eileen Yam for their contribution to the development of this conceptual framework, Sherry Hutchinson for graphics support, and Rebecca Brodsky for her review of the manuscript. We also want to thank our research partners at the University of Zimbabwe Clinical Trials Research Centre (Dr. Nyaradzo Mgodi, PI) and at Wits Reproductive Health and HIV Institute (Dr. Thesla Palanee-Phillips) for their collaboration on pilot DPP acceptability and adherence studies, and the members and advisors of the CIFF DPP Consortium who have contributed to our thinking about the value of this technology.

integration of family planning into HIV treatment services. Stud Fam Plann. (2017) 48:153-77. doi: 10.1111/sifp.12018

6. Kangudie DM, Guidigbi H, Mensah S, Bala AA, Delate R. Effective integration of sexual reproductive health and HIV prevention, treatment, and care services across sub-Saharan Africa: where is the evidence for program implementation? Reprod Health. (2019) 16(Suppl. 1):56. doi: 10.1186/s12978-019-0709-6

7. Warren CE, Mayhew SH, Hopkins J. The current status of research on the integration of sexual and reproductive health and HIV services: the current status of SRH-HIV integration research. Stud Fam Plann. (2017) 48:91-105. doi: 10.1111/sifp.12024

8. Ravindran TKS, Govender V. Sexual and reproductive health services in universal health coverage: a review of recent evidence from lowand middle-income countries. Sex Reprod Health Matters. (2020) 28:1779632. doi: 10.1080/26410397.2020.1779632

9. Romano J, Manning J, Hemmerling A, McGrory E, Young Holt B. Prioritizing multipurpose prevention technology development and investments using a target product profile. Antiviral Res. (2013) 100:S32-8. doi: 10.1016/j.antiviral.2013.09.016 
10. Tolley EE, Morrow KM, Owen DH. Designing a multipurpose technology for acceptability and adherence. Antiviral Res. (2013) 100:S54-9. doi: 10.1016/j.antiviral.2013.09.029

11. Harrison PF, Hemmerling A, Romano J, Whaley KJ, Young Holt B. Developing multipurpose reproductive health technologies: an integrated strategy. AIDS Res Treat. (2013) 2013:1-15. doi: 10.1155/2013/790154

12. Fernández-Romero JA, Deal C, Herold BC, Schiller J, Patton $\mathrm{D}$, Zydowsky $\mathrm{T}$, et al. Multipurpose prevention technologies: the future of HIV and STI protection. Trends Microbiol. (2015) 23:429-36. doi: 10.1016/j.tim.2015.02.006

13. Romano J, Van Damme L, Hillier S. The future of multipurpose prevention technology product strategies: understanding the market in parallel with product development. BJOG Int J Obstet Gynaecol. (2014) 121:15-8. doi: 10.1111/1471-0528.12850

14. Plagianos M, Kallianes V, Savel C, Pickett J, Guthrie K, Martinez C, et al. End-user opinions about the future of HIV prevention: results from a global internet survey about Multipurpose Prevention Technologies (MPTs). In: HIV Res Prevention Conference. Madrid (2018). Available online at: https://www. professionalabstracts.com/hivr4p2018/program-hivr4p2018.pdf.

15. van der Straten A, Agot K, Ahmed K, Weinrib R, Browne EN, Manenzhe K, et al. The Tablets, Ring, Injections as Options (TRIO) study: what young African women chose and used for future HIV and pregnancy prevention. J Int AIDS Soc. (2018) 21:e25094. doi: 10.1002/jia2.25094

16. IPSOS. Assessing the Potential of MPTs in South Africa, Uganda and Nigeria. IPSOS (2014). Available online at: https://www.theimpt.org/resource-results/ 35-reports/202-assessing-the-potential-of-mpts-in-south-africa-ugandaand-nigeria (accessed March 18, 2021)

17. Beksinska M, Greener R, Smit J, Maphumulo B, Mphili N, Kilbourne-Brook $\mathrm{M}$, et al. A randomized crossover study evaluating the use and acceptability of the SILCS diaphragm compared to vaginal applicators for vaginal gel delivery. AIDS Behav. (2018) 22:127-32. doi: 10.1007/s10461-017-1913-4

18. Laborde ND, Pleasants E, Reddy K, Atujuna M, Nakyanzi T, Chitukuta $\mathrm{M}$, et al. Impact of the dapivirine vaginal ring on sexual experiences and intimate partnerships of women in an HIV prevention clinical trial: managing ring detection and hot sex. AIDS Behav. (2018) 22:43746. doi: 10.1007/s10461-017-1977-1

19. Peasant C, Sullivan TP, Weiss NH, Martinez I, Meyer JP. Beyond the syndemic: condom negotiation and use among women experiencing partner violence. AIDS Care. (2017) 29:516-23. doi: 10.1080/09540121.2016.1224296

20. Exavery A, Kanté AM, Jackson E, Noronha J, Sikustahili G, Tani K, et al. Role of condom negotiation on condom use among women of reproductive age in three districts in Tanzania. BMC Public Health. (2012) 12:1097. doi: 10.1186/1471-2458-12-1097

21. Moore L, Beksinska M, Rumphs A, Festin M, Gollub EL. Knowledge, attitudes, practices and behaviors associated with female condoms in developing countries: a scoping review. Open Access J Contracept. (2015) 6:12542. doi: 10.2147/OAJC.S55041

22. IMPT for Reproductive Health. MPT Product Development Database. Available online at: https://mpts101.org/ (accessed march 18, 2021).

23. Fernandes T, Baxi K, Sawarkar S, Sarmento B, das Neves J. Vaginal multipurpose prevention technologies: promising approaches for enhancing women's sexual and reproductive health. Expert Opin Drug Deliv. (2020) 17:379-93. doi: 10.1080/17425247.2020.1728251

24. Brady M, Manning J. Lessons from reproductive health to inform multipurpose prevention technologies: don't reinvent the wheel. Antiviral Res. (2013) 100:S25-31. doi: 10.1016/j.antiviral.2013.09.019

25. United Nations, Department of Economic and Social Affairs, Population Division. Contraceptive Use by Method 2019: Data Booklet (ST/ESA/SER.A/435). United Nations (2019).

26. Begg L, Brodsky R, Friedland B, Mathur S, Sailer J, Creasy G. Estimating the market size for a dual prevention pill: adding contraception to preexposure prophylaxis (PrEP) to increase uptake. BMJ Sex Reprod Health. (2020) 1-7. doi: 10.1136/bmjsrh-2020-200662

27. Mugwanya K, Pintye J, Kinuthia J, Abuna F, Lagat H, Serede M, et al. Uptake of PrEP within clinics providing integrated family planning and PrEP services: results from a large implementation program in Kenya. In: Oral Abstract Presentation (TUAC0304). Amsterdam: (2018).
28. Gombe M, Mangwendeza Y, Ncube G, Zwangobani N, Prust M, Cakouros B, et al. Integrating oral HIV pre-exposure prophylaxis (PrEP) in a public family planning facility and youth center to inform national roll out in Zimbabwe. In: Oral Abstract Presentation (TUAC0307LB). Amsterdam: (2018).

29. Celum CL, Delany-Moretlwe S, Baeten JM, Straten A, Hosek S, Bukusi EA, et al. HIV pre-exposure prophylaxis for adolescent girls and young women in Africa: from efficacy trials to delivery. J Int AIDS Soc. (2019) 22(Suppl. 4):e25298. doi: 10.1002/jia2.25298

30. Amico KR, Wallace M, Bekker LG, Roux S, Atujuna M, Sebastian E, et al. Experiences with HPTN 067/ADAPT study-provided open-label PrEP among women in Cape Town: facilitators and barriers within a mutuality framework. AIDS Behav. (2017) 21:1361-75. doi: 10.1007/s10461-016-1458-y

31. Calabrese S, Dovidio J, Tekeste M, Taggart T, Galvao R, Safon C, et al. HIV pre-exposure prophylaxis stigma as a multidimensional barrier to uptake among women who attend planned parenthood. J Int AIDS Soc. (2018) 79:46-53. doi: 10.1097/QAI.0000000000001762

32. Corneli A, Perry B, McKenna K, Agot K, Ahmed K, Taylor J, et al. Participants' explanations for non-adherence in the FEM-PrEP clinical trial. J Int AIDS Soc. (2016) 71:452-61. doi: 10.1097/QAI.0000000000000880

33. Jani N, Mathur S, Kahabuka C, Makyao N, Pilgrim N. Relationship dynamics and anticipated stigma: key considerations for PrEP use among Tanzanian adolescent girls and young women and male partners. PLOS ONE. (2021) 16:e0246717. doi: 10.1371/journal.pone.0246717

34. Kyongo J, Kiragu M, Karuga R, Ochieng C, Ngunjiri A, Wachihi C, et al. How long will they take it? Oral pre-exposure prophylaxis (PrEP) retention for female sex workers, men who have sex with men and young women in a demonstration project in Kenya. In: Oral abstract presentation (WEAE0403). Amsterdam: (2018).

35. Population Council. Developing an Oral Contraceptive and HIV-Prevention Method, in a Single Pill. Available online at: https://www.popcouncil.org/ research/developing-an-oral-contraceptive-and-hiv-prevention-methodin-a-single-pill (accessed March 18, 2021).

36. Simmons R, Hall P, Diaz J, Diaz M, Fajans P, Satia J. The strategic approach to contraceptive introduction. Stud Fam Plann. (1997) 28:79. doi: 10.2307/2138111

37. Rosen RK, van den Berg JJ, Vargas SE, Senocak N, Shaw JG, Buckheit RW, et al. Meaning-making matters in product design: users' sensory perceptions and experience evaluations of long-acting vaginal gels and intravaginal rings. Contraception. (2015) 92:596-601. doi: 10.1016/j.contraception.2015.08.007

38. Weinrib R, Minnis A, Agot K, Ahmed K, Owino F, Manenzhe K, et al. Endusers' product preference across three multipurpose prevention technology delivery forms: baseline results from young women in Kenya and South Africa. AIDS Behav. (2018) 22:133-45. doi: 10.1007/s10461-017-1911-6

39. Patel SK, Rohan LC. On-demand microbicide products: design matters. Drug Deliv Transl Res. (2017) 7:775-95. doi: 10.1007/s13346-017-0385-4

40. Ajzen I. The theory of planned behavior: frequently asked questions. Hum Behav Emerg Technol. (2020) 2:314-24. doi: 10.1002/hbe2.195

41. Mathur S, Pilgrim N, Pulerwitz J. PrEP introduction for adolescent girls and young women. Lancet HIV. (2016) 3:e4068. doi: 10.1016/S2352-3018(16)30115-1

42. Mensch BS, van der Straten A, Katzen LL. Acceptability in microbicide and PrEP trials: current status and a reconceptualization. Curr Opin HIV AIDS. (2012) 7:534. doi: 10.1097/COH.0b013e3283590632

43. Merkatz RB, Plagianos M, Hoskin E, Cooney M, Hewett PC, Mensch BS. Acceptability of the Nestorone ${ }^{\circledR} /$ ethinyl estradiol contraceptive vaginal ring: development of a model; implications for introduction. Contraception. (2014) 90:514-21. doi: 10.1016/j.contraception.2014.05.015

44. MacQueen K, Cates W Jr. The multiple layers of prevention science research. Am J Prev Med. (2005) 28:491-5. doi: 10.1016/j.amepre.2005.02.020

45. Lusti-Narasimhan M, Collins L, Hopkins J. Lessons learnt from sexual and reproductive health and HIV linkages for multipurpose prevention technology service delivery. BJOG Int J Obstet Gynaecol. (2014) 121:8791. doi: 10.1111/1471-0528.12845

46. Kriel Y, Milford C, Cordero J, Suleman F, Beksinska M, Steyn P, et al. Male partner influence on family planning and contraceptive use: perspectives from community members and healthcare providers in KwaZulu-Natal, South Africa. Reprod Health. (2019)16:89. doi: 10.1186/s12978-019-0749-y 
47. Miller L, Morar N, Kapiga S, Ramjee G, Hayes R. Prevention, partners, and power imbalances: women's views on how male partners affected their adherence to vaginal microbicide gels during HIV Prevention Trials in Africa. J Acquir Immune Defic Syndr. (2020) 85:458-65. doi: 10.1097/QAI.0000000000002463

48. Kelly CA, Friedland BA, Morar NS, Katzen LL, Ramjee G, Mokgatle $\mathrm{MM}$, et al. To tell or not to tell: male partner engagement in a Phase 3 microbicide efficacy trial in South Africa. Cult Health Sex. (2015) 17:100420. doi: 10.1080/13691058.2015.1030451

49. Polis CB, Hussain R, Berry A. There might be blood: a scoping review on women's responses to contraceptive-induced menstrual bleeding changes. Reprod Health. (2018) 15:114. doi: 10.1186/s12978-01 8-0561-0

50. Callahan RL, Brunie A, Mackenzie ACL, Wayack-Pambè M, Guiella G, Kibira SPS, et al. Potential user interest in new long-acting contraceptives: results from a mixed methods study in Burkina Faso and Uganda. PLoS ONE. (2019) 14:e0217333. doi: 10.1371/journal.pone. 0217333
51. Delany-Moretlwe S, Mullick S, Eakle R, Rees H. Planning for HIV preexposure prophylaxis introduction: lessons learned from contraception. Curr Opin HIV AIDS. (2016) 11:87-93. doi: 10.1097/COH.0000000000000221

Disclaimer: The views and opinions expressed in this paper are those of the authors and not necessarily the views and opinions of CIFF or NIMH.

Conflict of Interest: The authors declare that the research was conducted in the absence of any commercial or financial relationships that could be construed as a potential conflict of interest.

Copyright $\odot 2021$ Friedland, Mathur and Haddad. This is an open-access article distributed under the terms of the Creative Commons Attribution License (CC BY). The use, distribution or reproduction in other forums is permitted, provided the original author(s) and the copyright owner(s) are credited and that the original publication in this journal is cited, in accordance with accepted academic practice. No use, distribution or reproduction is permitted which does not comply with these terms. 